\title{
Studien über Selensäure und selensaure Salze;
}

von Dr. v. Gerichten.

(Eingelaufen den 22. Mai 1873.)

Im Laboratorium von Herrn Prof. Dr. Hilge $r$ in Erlangen wurde diese Arbeit ausgeführt und besonders folgenden Hauptpunkten entgegengearbeitet : 1) einen sicheren, mit wenig Verlust verbundenen Weg zur Reindarstellung der Selensäure aufzufinden, 2) Selensäureanhydrid darzustellen, 3) selensaure Doppelsalze zu untersuchen und zu messen, 4) Selensäure mit Schwefelsäure in Doppelsalzen auf jede mögliche Art zu combiniren und die erhaltenen Producte, selenschwefelsaure und schwefelselensaure Salze, zu messen.

Zur Darstellung der Selensäure werden aufser der von Mitscherlich eingeführten verschiedene Methoden angegeben, von denen die von v. Hauer *) wohl die beste, wenn auch nicht die einfachste ist, die jedoch alle den Nachtheil haben, dafs sie reines Selen als Ausgangspunkt annehmen, wenn nicht ein gröfserer Umweg gemacht werden sollte. Der ersten und einfachsten Methode von Mitscherlich anschliefsend, wurde, um aus schwefelhaltigem Selen reine Selensäure zu erhalten, folgendes Verfahren als empfehlenswerth erachtet : Oxydation des Selens mittelst Salpetersäure zu seleniger Säure, Zusatz von Kali, Einleiten von Chlor, so lange als Zinnchlorür in einer kleinen Probe der Flüssigkeit noch eine rothe Fällung erzeugt. Sodann Fällen der Selensäure als Barytsalz, Zersetzen des letzteren mit wenig Ueber-

*) Wien. acad. Ber. 89, 399 ; Jahresber. f. Chem. u. s. w. f. $1860,85$. 
schufs von kohlensaurem Kali in der Kälte. Mitgefällter schwefels. Baryt bleibt unzersetzt. Hierfür sprechen folgende Zahlenangaben :

24,23 Grm. selensaurer Baryt gaben nach dem Behandeln mit kohlensaurem Kali und Wiederausfällen des ersteren aus der vorher natürlich mit Salpetersäure neutralisirten Lösung durch Chlorbaryum 23,81 selensauren Baryt; 0,64 selensaurer Baryt gemengt mit 0,78 schwefelsaurem Baryt gaben nach dem nämlichen Verfahren wieder 0,59 selensauren Baryt.

Man sieht, es ist diese Methode der Trennung von Selensäure und Schwefelsäure keineswegs zur quantitativen Analyse geeignet, wie H. Rose *) ganz richtig betont, aber in unserem Falle immer noch dem Zwecke hinreichend entsprechend. - Hierauf Ausfällen der Selensäure durch Bleisalzlösung, Decantiren, Filtriren und Zersetzung des selensauren Bleioxyds durch Schwefelwasserstoff.

Eine Schattenseite des Verfahrens durch Chlor zu oxydiren und die Selensäure durch Barytsalz zu fällen wäre, dafs der selensaure Baryt in Wasser weniger unlöslich ist, als der schwefelsaure (H. Ros $\mathrm{e}^{* *}$ ). Jedoch ist es möglich, nach öfterem Decantiren und tüchtigem Auswaschen des selensauren Barytniederschlags aus dem Filtrate durch geringe Concentration allen selensauren Baryt wieder zu gewinnen, so dafs nach der Filtration im Filtrate keine Spur von Selensäure mehr nachweisbar ist. Was die von Wohlwill ***) erwähnte unvollständige Zersetzung des Bleiniederschlags durch Schwefelwasserstoff betrifft, so habe ich mich überzeugt, dafs im Schwefelbleirückstand, allerdings nach dem Durchleiten eines sehr starken Stroms Schwefelwasserstoff, keine Spur von

*) Zeitschr. f. anal. Chem. 1, 73.

**) Pogg. Ann. 95, 426; Jahresber. f. Chem. u. s. w. f. 1855, 276.

***) Inauguraldissert., Göttingen 1860; diese Ann. 114, 169 ; Jahresber. f. Chem. u. s. w. f. $1860,89.90$. 
Selensäure mehr nachzuweisen war. Es sei nun noch kurz der bis jetzt angegebenen anderen Methoden Erwähnung gethan. a. Mitscherlich giebt an, mit Salpeter selenigsaures Kali zu schmelzen und mit Blei zu fällen, den Bleiniederschlag sodann mit Schwefelwasserstoff zu zersetzen. Die so erhaltene Selensäure hinterläfst immer beim Verdampfen auf Platinblech einen Rückstand; demnach wäre noch salpetersaures Salz vorhanden, und man hätte die Säure etwa mit kohlensaurem Kupferoxyd zu neutralisiren und das so erhaltene selensaure Kupfersalz mit Schwefelwasserstoff zu zersetzen. b. v. Hauer löst das mit Salpeter geschmolzene selenigsaure Salz in Wasser, versetzt mit salpetersaurem Kalk und gewinnt das selensaure Salz durch Concentration, zersetzt letzteres mittelst oxalsauren Cadmiums und behandelt das selensaure Cadmium mit Schwefelwasserstoff. c. Wohlwill gründet seine Methode auf die Oxydation des selenigsauren Kupferoxyds durch Chlor, Abscheiden des selensauren Salzes durch Weingeist und Zersetzung desselben durch Schwefelwasserstoff. Jedoch ist leicht ersichtlich, dafs diese Methode schon grofse Uebung erfordert, da es ohne dieselbe kaum möglich ist, vollständig von Kupferchlorid freies selensaures Salz zu fällen. Trotzdem wäre diese Darstellungsweise eine ganz vortreffliche, wenn nicht noch $\mathrm{T}$ ops ö $\mathrm{e}^{*}$ ) nachgewiesen hätte, dafs die Zersetzung des selensauren Kupferoxyds durch Schwefelwasserstoff oder vielmehr das Auswaschen des entstandenen Schwefelkupfers niemals ohne Oxydation vor sich geht, so dafs man selbst bei ungenügendem Auswaschen immer noch Schwefelsäure im Filtrate nachweisen kann. Dasselbe ist auch noch bei der Methode von Mitscherlich zu bemerken, der ja ebenfalls den Weg zur Zersetzung des

*) Inauguraldiss. : Kryst. Kem. Undersogelser ov de selens. Salte. Kjobenhaven 1870; Tidsskr. for Chem. og Phys. 1870, 9. 245. 
Kupfersalzes einschlägt. d. Zuletzt sei noch eines Verfahrens Erwähnung gethan, dafs sich auf Zersetzung und Oxydation des selenigsauren Silberoxyds durch Brom gründet.

Zur Darstellung des Selensäureanhydrids sind bis jetzt folgende Versuche gemacht worden. F a b i an hat dadurch, dafs er concentrirte wässerige Selensäure wiederholt möglichst heifs bei 240 bis $260^{\circ} \mathrm{C}$. neben Vitriolöl unter die Glocke der Luftpumpe brachte und evacuirte, eine Selensäure von 2,627 spec. Gew. mit 85,46 pC. Anhydrid erhalten. Eine gröfsere Concentration der Säure ist bis jetzt nicht erreicht worden; ja nicht einmal das Selensäurehydrat ist erreicht worden, welches einen Gehalt von 87,63 Anhydrid besitzen müfste. Es wurde nun der Versuch angestellt, ob Selensäureanhydrid nicht durch die sogenannte Contactwirkung des Platinschwammes zu erhalten wäre; zu dem Ende wurde die eine Hälfte einer kurzen Verbrennungsröhre zum vierten Theil ungefähr mit in Asbest fein zertheiltem Platinschwamm gefüllt, in die Mitte der andern Hälfte reines Selen gebracht und nun die ganze Röhre in Verbindung gesetzt mit einem mit Sauerstoff gefüllten Gasometer; der Sauerstoff wurde zuerst durch eine mit Schwefelsäure gefüllte $W$ o u lf e'sche Flasche, dann durch ein System von mit wasserfreiem Kupfervitriol, Bimsstein und Chlorcalcium gefültten Röhren geleitet und dann erst gelangte er in die eigentliche Verbrennungsröhre. Der Platinschwamm wurde glühend gemacht und sodann das Selen gelinde erwärmt. Es zerfliefst, fängt an zu brennen und an den kälteren Stellen vor dem Platinschwamm setzt sich allmälig eine weifse Schicht von seleniger Säure an, die nun unter fortwährendem Zuleiten von Sauerstoff durch den glühenden Platinschwamm getrieben wird. Auf diese Weise ist es gelungen, in einer vorgeschobenen Vorlage einem dem Schwefelsäureanhydrid ähnlichen weifsen Anflug zu erhalten, der in Wasser unter Zischen sich löste, jedoch keineswegs reines Selensäureanhydrid 
war, denn in der wässerigen Lösung gab Zinnchlorür sofort eine rothe Fällung, ein Beweis für das Vorhandensein seleniger Säure. Dagegen wurde in eben der Lösung mit Chlorbaryum ein Niederschlag erhalten, der in Säuren sich nicht löste und beim Kochen mit Salzsäure einen entschiedenen Geruch nach frei werdendem Chlor bemerkbar machte, ein Beweis für das Vorhandensein von Selensäure. Jedoch wurde bei einem zweiten Versuch ein entschieden negatives Resultat erhalten, indem sich in dem durch Platinschwamm geleiteten Producte nur selenige Säure vorfand und in der sauren Lösung kein Niederschlag von Chlorbaryum erhalten wurde. Es scheint demnach sich allerdings durch ,Contactwirkung“ Selensäureanhydrid ganz analog dem Schwefelsäureanhydrid zu bilden, jedoch glaube ich, dafs sich letzteres sofort nach der Bildung wieder theilweise zersetzt, was natürlich ist bei den hohen Hitzegraden, die angewandt werden müssen. Jedenfalls verdienten diese Versuche eine weitere Beachtung und statt des etwas kostspieligen Platinschwamms könnte man vielleicht Chromoxyd oder Eisenoxyd verwenden und ein auf solche Weise erhaltenes selensaures Eisenoxyd und Chromoxyd würde eben so für die Existenz des Selensäureanhydrids sprechen, als dessen Reindarstellung selber.

Die selensauren Salze sind, wie bekannt, im Allgemeinen mit den schwefelsauren und mangansauren isomorph und eben so sollten sie es sein mit den molybdänsauren und wolframsauren, ja sogar mit den eisensauren entsprechend der allgemeinen Formel $\mathrm{M}_{2} \mathrm{RO}_{4}$.

Diesen Isomorphismus der selensauren Salze mit den schwefelsauren nachzuweisen, gab wohl zu einer Zeit, als der Chemiker den genau gekannten Gesetzen des Homöomorphismus mit mehr Recht Achtung zollte, als der heutige Chemiker den unsicheren Andeutungen einer sogenannten Werthigkeit, den ersten Impuls zur genaueren Untersuchung der selensauren 
Salze, und so hatte denn auch Mitscherlich selber schon in dieser Richtung den beiden späteren Forschern v. Hau er*) und $\mathrm{Wohlwill}^{* *}$ ) vorgearbeitet. In neuester Zeit hat Haldor Topsö e**) die selensauren Salze und Doppelsalze insbesondere einer genaueren krystallographischen Untersuchung unterworfen, und so sei es mir denn vergönnt, aus diesem Material vorerst eine übersichtliche Zusammenstellung der bis jetzt untersuchten selensauren Salze zu geben, bevor ich zu den von mir dargestellten Doppelsalzen übergehe.

$\mathrm{K}_{2} \mathrm{SeO}_{4}$, Pogg. Ann. 18, 168. $1830 ; \mathbf{3 9}, 198$.

$\mathrm{HKSeO}_{4}$, Pogg. Ann. 18, 173; 39, 198. H. Top söe 13.

$\mathrm{Na}_{2} \mathrm{SeO}_{4}$, Pogg. Ann. 11, 325; v. Hauer, Sitzungsber. 89, 299.

$\mathrm{Li}_{2} \mathrm{SeO}_{4}+\mathrm{H}_{2} \mathrm{O}$, H. To psöe 15 .

$\left.\left(\mathrm{NH}_{4}\right)_{2} \mathrm{SeO}_{4}\right)$ v. Lang, Wien. acad. Ber. 45, 109.1862.

$\left.\mathrm{HNH}_{4} \mathrm{SeO}_{4}\right\}$ H. Tops öe 15.

$\mathrm{MgSeO}_{4}+6 \mathrm{H}_{2} \mathrm{O}_{\text {/ }}$ ( Pogg. Anl. 11, 327.

$\mathrm{CaSeO}_{4}+2 \mathrm{H}_{2} \mathrm{O}$, v. $\mathrm{Hauer}$, Wien. acad. Ber. $\mathbf{3 9}$, 309. 837 .

$\mathrm{BaSeO}_{4}$, H. Ros e, Pogg. Ann. 95, 426.

$\mathrm{NiSeO}_{4}+6 \mathrm{H}_{2} \mathrm{O}$, Mitscherlich, Pogg. Ann. I2, $144 ; \mathrm{H}$. Topsöe 13.

$\mathrm{NiSeO}_{4}+7 \mathrm{H}_{2} \mathrm{O}$, Marignac, Recherches sur les formes crist. p. 42 ; Ann. min. [5] D, 27 ; v. Hauer, Wien. acad. Ber. 89, 305.

$\mathrm{CoSeO}_{4}+5 \mathrm{H}_{2} \mathrm{O}$, Mitseherlich, Pogg. Ann. 11, 330; H. Topsöe 22 .

$\mathrm{CoSeO}_{4}+6 \mathrm{H}_{2} \mathrm{O}, \mathrm{H}$. Top söe 28 .

$\mathrm{CoSeO}_{4}+7 \mathrm{H}_{2} \mathrm{O}$, H. Topsöe 32 .

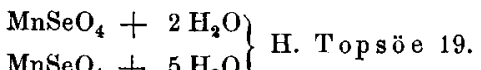

*) Wien. acad. Ber. 89, 299. 837. 1860; im Auszug : Chem. Centralbl. 1860,883 .

**) Inauguraldiss., Göttingen 1860; diese Ann. 114, 169; Jahresber. f. Chem. u. s. w. f. 1860,8990 .

**) Inauguraldiss. kryst. ov de selens. Salte. Kjobenhaven 1860; Tidsskr. for Chem. og Phys. 1870, 9. 245. 
$\mathrm{YSeO}_{4}+5 \mathrm{H}_{2} \mathrm{O}$, Jahresber. f. Chem. u. s. w. f. $1860,203$.

$\mathrm{FeSeO}_{4}+5 \mathrm{H}_{2} \mathrm{O}, \mathrm{Woh} \mathrm{will}_{\mathrm{w}}$, diese Ann. 14, 169 .

$\mathrm{FeSeO}_{4}+7 \mathrm{H}_{2} \mathrm{O}$, Wohlwill, Inauguraldiss. S. 47 ; H. Tops ö 33 .

$\mathrm{ZnSeO}_{4}+5 \mathrm{H}_{2} \mathrm{O}$, Pogg. Ann. 11, 329; H. Topsöe 24.

$\mathrm{ZnSeO}_{4}+6 \mathrm{H}_{2} \mathrm{O}$, H. Topsöe 30 .

$\mathrm{Tl}_{2} \mathrm{SeO}_{4}, \mathrm{Kuhlmann}$, Bull. soc. chim. [2] 1, 330; O ettinger,

On the comb. of Thallium. Berlin 1864, 29; v. Lang, Wien. acad. Ber. 55, 415. 1867.

$\mathrm{HTlSeO}_{4}+3 \mathrm{H}_{2} \mathrm{O}$, Oettinger.

$\mathrm{CdSeO}_{4}$, v. Hauer, Wien. acad. Ber. 89, 299; H. Top sö e 18. $\mathrm{PbSeO}_{4}+\mathrm{Pb}(\mathrm{OH})_{2}$, Barfoed.

$\mathrm{PbSeO}_{4}$, Mitscherlich, Schafarik, Wien. acad. Ber. 48, 256; Chem. Centralbl. 1863, 580.

$\mathrm{CuSeO}_{4}+5 \mathrm{H}_{2} \mathrm{O}$, Mittcherlich, Pogg. Ann. In, 330; H. Topsöe 25.

$\mathrm{Ag}_{2} \mathrm{SeO}_{4}, \mathrm{Mitscherlieh}$.

So weit geht unsere Kenntnifs der einfachen selensauren Salze. Sie sind sämmtlich mit den entsprechenden schwefelsauren isomorph, unterscheiden sich jedoch von letzteren wieder durch ein gröfseres spec. Volumen, sodann dadurch, dafs die selensauren Salze unter gleichen Bedingungen mit geringeren Wassermengen krystallisiren, als die schwefelsauren, und um die gleichen Wassermengen aufzunehmen, wie diese, eine weit niedrigere Krystallisationstemperatur erfordern. Von den Salzen, die H. Topsö e zum Beweise dieser Thatsache anführt, die übrigens von v. Ha u er *) schon 1866 ebenfalls beobachtet wurden, seien hier nur die Mangansalze erwähnt. Das selensaure Manganoxydul krystallisirt bei $0^{\circ}$ in der gewöhnlichen Form mit $5 \mathrm{H}_{2} \mathrm{O}$ und bei 30 bis $40^{\circ} \mathrm{C}$. mit $2 \mathrm{H}_{2} \mathrm{O}$, das schwefelsaure bei 4 bis $6^{0} \mathrm{C}$. mit $7 \mathrm{H}_{2} \mathrm{O} * *$ ), bei 30 bis $40^{\circ}$ C. mit $4 \mathrm{H}_{2} \mathrm{O} * * *$ ).

*) Wien. acad. Ber. 54, 1866.

**) Regnault, Ann. chim. et phys. [2] 6, 200.

***) Marig n a c, Recherches sur les formes crist. etc. 1855, p. 38 . 
Endich ist noch als Unterschied zwischen selensauren und schwefelsauren Salzen hervorzuheben, dafs das Verhältnifs des Axenwinkels zu den beiden anderen Winkeln bei diesen kleiner ist, als bei jenen. H. Topsöe 5 und 64 .

$\mathrm{Ha}$ Id or Topsö e hat ferner folgende Doppelsalze, von denen v. Hau er und Wohlwill die meisten analysirt haben, krystallographisch untersucht :

$\begin{array}{ll}\mathrm{CdSeO}_{4} \cdot\left(\mathrm{NH}_{4}\right)_{2} \mathrm{SeO}_{4}+2 \mathrm{H}_{2} \mathrm{O} & \mathrm{CuSeO}_{4} \cdot\left(\mathrm{NH}_{4}\right)_{2} \mathrm{SeO}_{4}+6 \mathrm{H}_{2} \mathrm{O} \\ \mathrm{MnSeO}_{4} \cdot \mathrm{H}_{2} \mathrm{SeO}_{4}+2 \mathrm{H}_{2} \mathrm{O} & \mathrm{MnSeO}_{4} \cdot\left(\mathrm{NH}_{4}\right)_{2} \mathrm{SeO}_{4}+6 \mathrm{H}_{2} \mathrm{O} \\ \mathrm{ZnSeO}_{4} \cdot \mathrm{H}_{2} \mathrm{SeO}_{4}+2 \mathrm{H}_{2} \mathrm{O} & \mathrm{CaSeO}_{4} \cdot\left(\mathrm{NH}_{4}\right)_{2} \mathrm{SeO}_{4}+6 \mathrm{H}_{2} \mathrm{O} \\ \mathrm{MgSeO}_{4} \cdot\left(\mathrm{NH}_{4}\right)_{2} \mathrm{SeO}_{4}+6 \mathrm{H}_{2} \mathrm{O} & \mathrm{MgSeO}_{4} \cdot \mathrm{K}_{2} \mathrm{SeO}_{4}+6 \mathrm{H}_{2} \mathrm{O} \\ \mathrm{NiSeO}_{4} \cdot\left(\mathrm{NH}_{4}\right)_{2} \mathrm{SeO}_{4}+6 \mathrm{H}_{2} \mathrm{O} & \mathrm{CoSeO}_{4} \cdot \mathrm{K}_{2} \mathrm{SeO}_{4}+6 \mathrm{H}_{2} \mathrm{O} \\ \mathrm{CoSeO}_{4} \cdot\left(\mathrm{NH}_{4}\right)_{2} \mathrm{SeO}_{4}+6 \mathrm{H}_{2} \mathrm{O} & \mathrm{FeSeO}_{4} \cdot \mathrm{K}_{2} \mathrm{SeO}_{4}+6 \mathrm{H}_{2} \mathrm{O} \\ \mathrm{FeSeO}_{4} \cdot\left(\mathrm{NH}_{4}\right)_{2} \mathrm{SeO}_{4}+6 \mathrm{H}_{2} \mathrm{O} & \mathrm{ZnSeO}_{4} \cdot \mathrm{K}_{2} \mathrm{SeO}_{4}+6 \mathrm{H}_{2} \mathrm{O} \\ \mathrm{ZnSeO}_{4} \cdot\left(\mathrm{NH}_{4}\right)_{2} \mathrm{SeO}_{4}+6 \mathrm{H}_{2} \mathrm{O} & \mathrm{CuSeO}_{4} \cdot \mathrm{K}_{2} \mathrm{SeO}_{4}+6 \mathrm{H}_{2} \mathrm{O} .\end{array}$

Aufserdem hat v. $\mathrm{Hau}$ er Doppelsalze beschrieben $\mathrm{CdSeO}_{4}$ $+\mathrm{K}_{2} \mathrm{SeO}_{4}+2 \mathrm{H}_{2} \mathrm{O}, \mathrm{NiSeO}_{4} \cdot \mathrm{K}_{2} \mathrm{SeO}_{4}+6 \mathrm{H}_{2} \mathrm{O}$. Hiervon beschrieb v. Rath (Pogg. Ann. 115, 485) die Krystallform. G. Werther hat dargestellt $\mathrm{ZnSeO}_{4}+\mathrm{Tl}_{2} \mathrm{SeO}_{4}+6 \mathrm{H}_{2} \mathrm{O}$.

Sodann seien noch die Selensäurealaune von Wohlwill berücksichtigt : $\left.\begin{array}{l}\mathrm{SeO}_{2} \\ \mathrm{SeO}_{2}\end{array}\right\}\left\{\begin{array}{l}\mathrm{Al}(\mathrm{Cr}) \\ \mathrm{K} .\left(\mathrm{Na} . \mathrm{NH}_{4}\right)\end{array}+12 \mathrm{H}_{2} \mathrm{O}\right.$. Endlich bespricht Wohlwill noch von ihm dargestellte selenschwefelsaure Doppelsalze :

$\mathrm{FeSO}_{4}+\mathrm{CuSeO}_{4}, \mathrm{MgSO}_{4}+\mathrm{CuSeO}_{4}, \mathrm{ZnSO}_{4}+\mathrm{CuSeO}_{4}$.

Jedoch wurde gerade diese Richtung der theilweisen Ersetzung der Schwefelsäure durch Selensäure in Doppelsalzen bis heute meines Wissens nicht weiter berücksichtigt. So war denn mein Augenmerk hauptsächlich nach dieser Seite gerichtet und es wurden schwefelselensaure Doppelsalze einerseits und selenschwefelsaure andererseits dargestellt und einer genauen Untersuchung unterworfen. 


\section{Alaune.}

1. Reihe $: \underset{\mathrm{SeO}_{2}}{\mathrm{SO}_{2}}\langle\}_{\mathbf{r}} \mathrm{R}+12 \mathrm{H}_{2} \mathrm{O}$. a. Schwefelselensaures Thonerde-Kali wurde erhalten durch Mischen der Lösungen von schwefelsaurer Thonerde und selensaurem Kali. Es bildet klare wasserhelle Octaëder von folgender Zusammensetzung :

$\begin{array}{lcc} & \text { Berechnet } & \text { Gefunden } \\ \mathrm{SO}_{3} & 24,09 & 23,81 \\ \mathrm{SeO}_{3} & 12,78 & 12,95 \\ \mathrm{HO} & 43,37 & 43,41 \\ \mathrm{KO} & 9,45 & 9,87 \\ \mathrm{Al}_{2} \mathrm{O}_{3} & 10,31 & 9,84\end{array}$

entsprechend der Formel $\mathrm{SeO}_{3} \mathrm{KO}+\left(\mathrm{SO}_{3}\right)_{3} \mathrm{Al}_{2} \mathrm{O}_{3}+24 \mathrm{aq}$.

Nimmt man saure schwefelsaure Thonerde zur Mischung mit selensaurem Kali, so scheint eine Umsetzung von Schwefelsäure nach der Selensäure des Kali's`stattzufinden, indem man einen Alaun erhält, in dem die Selensäure des Kalis theilweise durch Schwefelsäure ersetzt ist. Auf diese Weise ist es möglich, eine Reihe von Salzen zu erhalten, in denen procentweise Selensäure durch Schwefelsäure ersetzt ist.

Was die Analyse dieser und der nachfolgenden Salze anbelangt, so wurde sie immer in zwei für sich abgewogenen Theilen vorgenommen; im einen wurde Selen und Kali, im anderen die übrigen Bestandtheile bestimmt. Im ersten Falle wurde das Salz in Salzsäure gelöst und stark gekocht zur Reduction der Selensäure zu seleniger Säure und dann das Selen als solches mit doppelt-schwefligsaurem Ammoniak abgeschieden und auf einem bei $100^{\circ} \mathrm{C}$. getrockneten Filter gewogen; sodann wurde die Thonerde durch kohlensaures Ammoniak abgeschieden und das Kali nach den gewöhnlichen Methoden bestimmt. Im zweiten Falle kocht man das gewogene Salz eben so mit Salzsäure und versetzt mit Chlor- 
baryum. Der schwefelsaure Baryt wird rasch abfiltrirt und das Filtrat mit kohlens. Ammoniak versetzt; es fällt kohlensaurer Baryt und basisch-kohlensaure Thonerde. Der Niederschlag wird wie gewöhnlich mit Schwefelsäure zersetzt und im Filtrat des schwefelsauren Barytrückstandes die Thonerde bestimmt.

b. Schwefelselensaures Chromoxyd-Kali wurde in dem schwefelsauren Chromalaun ganz gleichen Octaëdern erhalten. Ferner schwefelselensaures Eisenoxyd- und Manganoxyd-Kali.

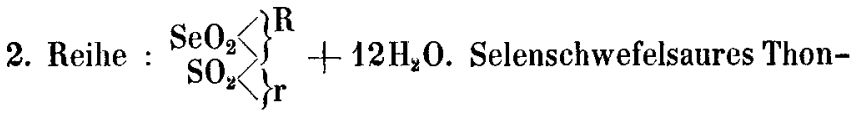
erde-Kali wurde in wasserhellen Octaëdern erhalten durch Mischen der Lösungen von selensaurer Thonerde mit schwefelsaurem Kali. Nach einiger Zeit schiefsen Octaëder an von der Zusammensetzung :

$\begin{array}{lcc} & \text { Berechnet } & \text { Gefunden } \\ \mathrm{SeO}_{\mathbf{8}} & 35,03 & 34,64 \\ \mathrm{SO}_{3} & 7,33 & 7,85 \\ \mathrm{HO} & 39,59 & 39,71 \\ \mathrm{KO} & 8,63 & - \\ \mathrm{Al}_{2} \mathrm{O}_{3} & 9,42 & 8,96\end{array}$

entsprechend der Formel $\mathrm{SO}_{3} \mathrm{KO}$. $\left(\mathrm{SeO}_{3}\right)_{3} \mathrm{Al}_{2} \mathrm{O}_{3}+24 \mathrm{aq}$.

In derselben Weise ist dargestellt der selenschwefelsaure Chromalaun und Eisenalaun. Die Krystalle der ganzen Gruppe unterscheiden sich durch Nichts von denen der gewöhnlichen Alaune und überall trifft man die so charakteristischen Formen, welche bekanntlich entstehen durch Verkürzung des Octaëders nach der hexaëdrischen Axe; eben so sind bei den hier beschriebenen selenschwefelsauren und schwefelsauren Chromoxydkalisalzen auch jene verzerrten, oft fragmentarischen Octaëderformen, wie sie bei dem gewöhnlichen Chromalaun vorkommen, gefunden worden.

Zur Uebersicht der untersuchten Salze möge folgende Zusammenstellung dienen, deren Anfangsglied der gewöhnliche 
Alaun, deren Endglied der von Wo h lwill dargestellte Selensäurealaun ist :

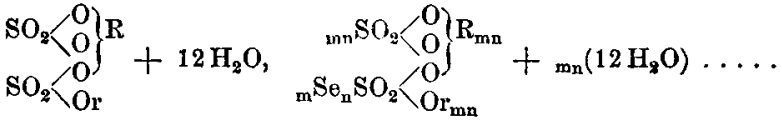

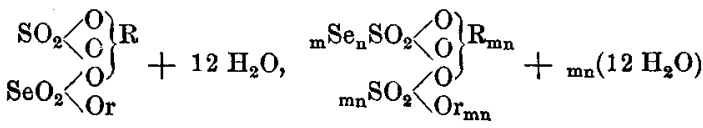

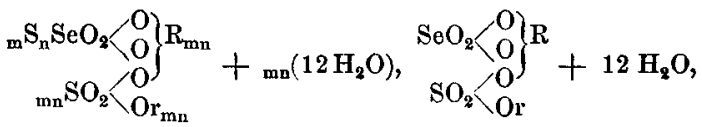

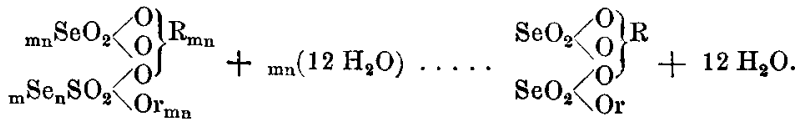

II. Doppelsalze der allgemeinen Formel $\mathrm{m}_{2} \mathrm{RO}_{4}$. $\mathbf{M R O}_{4}$ $+6 \mathrm{H}_{2} \mathrm{O}$.

1. Reihe : $\mathrm{MSO}_{4} \cdot \mathrm{m}_{2} \mathrm{SeO}_{4}+6 \mathrm{H}_{2} \mathrm{O}$. Schwefelselensaures Nickeloxydul-Kali wurde in prächtig grünen Krystallen des monoklinen Systems dargestellt durch Mischen der concentrirten Lösungen von schwefulsaurem Nickel mit selensaurem Kali. Auch hier ist, wie bei den entsprechenden Alaunen und allen folgenden Salzen, Rücksicht zu nehmen, dafs die Lösung von schwefelsaurem Nickel nicht, wie es zumeist der Fall ist, sauer reagire. Es resultirt sonst ein Salz, in dem die Selensäure des selensauren Kali's theilweise durch Schwefelsäure ersetzt ist. Was die procentische Zusammensetzung des Salzes anbelangt, so wurde bei der Analyse folgendes Resultat erhalten :

$\begin{array}{lcc} & \text { Berechnet } & \text { Gefunden } \\ \mathrm{SO}_{3} & 16,52 & 16,63 \\ \mathrm{SeO}_{3} & 26,30 & 26,84 \\ \mathrm{HO} & 22,29 & 22,26 \\ \mathrm{KO} & 19,45 & 20,10 \\ \mathrm{NiO} & 15,44 & 14,81\end{array}$

entsprechend der Formel $\mathrm{SO}_{3} \mathrm{NiO} . \mathrm{SeO}_{3} \mathrm{~K}_{2} \mathrm{O}+6 \mathrm{H}_{2} \mathrm{O}$.

Bei den monoklinen Krystallen ist $0 \mathrm{P}$ vorherrschend, wodurch der gewöhnlich tafelförmige Habitus bedingt ist. 
Ferner wurde beobachtet o $\mathrm{P}$ und das Klinodoma $\mathrm{P} \infty$, ebenso wie ein Hemiorthodoma $2 \mathrm{P} \infty$ oft deutlich mefsbar ausgebildet. Eben so wurde gefunden das klinodiagonale Flächenpaar $\infty \mathrm{P} \infty$.

$\infty \mathrm{P}: \infty \mathrm{P}=(110: \overline{1} 10) 106^{0} 32^{\prime},(110: 1 \overline{1} 0) 73^{0} 28^{\prime} .0 \mathrm{P}: \infty \mathrm{P}=$

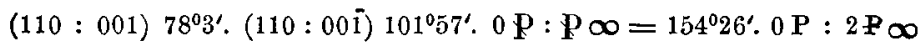
$=117^{0} 46^{\prime}$. ac $=75^{\circ} 7^{\prime}$. Spec. Gew. $=2,34$, spec. Volumen $=103,5$.

Die frischen Krystalle sind diamantglänzend und behalten ihre stark spiegelnden Flächen. Beim Lösen derselben in concentrirter Salzsäure, was behufs der Analyse öfters vorgenommen werden mufste, wurde die Beobachtung gemacht, dafs dadurch gleichsam eine Zersetzung des Doppelsalzes eintritt, indem sofort (natürlich in der Kälte) eine grüne Lösung des schwefelsauren Nickelsalzes stattfindet, während das selensaure Kali sämmtlich noch ungelöst bleibt, so dafs dasselbe als weifses Pulver beim Abschütten der grünen Lösung zurückbleibt. Dieses Verhältnifs könnte vielleicht interessante Aufschlüsse bieten über die Atomlagerung in diesen Doppelsalzen.

Das Krystallwasser geht erst bei $140^{\circ} \mathrm{C}$. vollständig fort, bei 100 bis $110^{\circ}$ ist nur ein Gewichtsverlust von etwa $18 \mathrm{pC}$. zu bemerken.

Ferner wurden dargestellt, derselben Untersuchung unterworfen und als isomorph befunden mit den entsprechenden rein selen- und rein schwefelsauren Doppelsalzen folgende schwefelselensaure Doppelsalze :

$$
\begin{aligned}
& \mathrm{CoSO}_{4} \cdot \mathrm{K}_{2} \mathrm{SeO}_{4}+6 \mathrm{H}_{2} \mathrm{O} \\
& \mathrm{FeSO}_{4} \cdot \mathrm{K}_{2} \mathrm{SeO}_{4}+6 \mathrm{H}_{2} \mathrm{O} \\
& \mathrm{ZnSO}_{4} \cdot \mathrm{K}_{2} \mathrm{SeO}_{4}+6 \mathrm{H}_{2} \mathrm{O} \\
& \mathrm{MnSO}_{4} \cdot \mathrm{K}_{2} \mathrm{SeO}_{4}+6 \mathrm{H}_{2} \mathrm{O} \\
& \mathrm{CdSO}_{4} \cdot \mathrm{K}_{2} \mathrm{SeO}_{4}+6 \mathrm{H}_{2} \mathrm{O} \\
& \mathrm{CuSO}_{4} \cdot \mathrm{K}_{2} \mathrm{SeO}_{4}+6 \mathrm{H}_{2} \mathrm{O} \\
& \mathrm{MgSO}_{4} \cdot \mathrm{K}_{2} \mathrm{SeO}_{4}+6 \mathrm{H}_{2} \mathrm{O} .
\end{aligned}
$$


Hiermit wäre auch diese Reihe geschlossen und ich gehe nun über zu den selenschwefelsauren Doppelsalzen der nämlichen altgemeinen Formel, in denen das $\mathrm{R}$ des M-Salzes durch Se ersetzt ist.

2. Reihe. $\mathrm{MSeO}_{4} \cdot \mathrm{m}_{2} \mathrm{SO}_{4}+6 \mathrm{H}_{2} \mathrm{O}$. Selenschwefelsaures Kupferoxyd-Kali wurde erhalten durch Mischung der betreffenden Lösungen. Es krystallisirt leicht aus und es ist auch hier wie bei den früher beschriebenen Salzen möglich, durch Selensäure allmälig erst die Schwefelsäure die an Kupferoxyd gebunden war, und dann die welche das Kaliumsulfat bildete, verdrängen zu lassen. Die quantitative Analyse des Salzes ergab :

$\begin{array}{lcc} & \text { Berechnet } & \text { Gefunden } \\ \mathrm{SeO}_{3} & 26,05 & 25,74 \\ \mathrm{SO}_{3} & 16,36 & 16,79 \\ \mathrm{HO} & 22,09 & 22,41 \\ \mathrm{KO} & 19,26 & - \\ \mathrm{CuO} & 16,24 & -\end{array}$

entsprechend der Formel $\mathrm{SeO}_{3} \mathrm{CuO} . \mathrm{SO}_{3} \mathrm{KO}+6 \mathrm{aq}$.

Das selenschwefelsaure Kupferoxydkali krystallisirt in schönen, wohlausgebildeten, lichtblauen, monoklinischen Combinationen oft tafelförmig, meist aber mehr säulenförmig mit starker Verlängerung des basischen Pinakoïds. Die Flächen sind meist klar spiegelnd, wenn das Salz frisch hergestellt ist, werden aber bald matt. Die Messungen, die an wohlausgebildeten Exemplaren vorgenommen wurden, ergaben Folgendes :

$\infty \mathrm{P}: \infty \mathrm{P}=(110: \overline{1} 10) 108^{\circ}(110: 1 \overline{1} 0) 72^{\circ} .0 \mathrm{P}: \infty \mathrm{P}=(110: 001)$ $79^{\circ} 81^{\prime}$ (110:001) 100052'. 0 P : P $\infty=153^{\circ} 30^{\prime}$. ac $=76^{\circ} 33^{\prime}$. Das selensaure Doppelsalz hat nach H. Topsöe ac $=76^{\circ} 41^{\prime}$.

Es ist demnach dieses Salz vollständig isomorph mit den selensauren Doppelsalzen und eben dieses ist auch bei den anderen Salzen dieser Reihe der Fall. Sämmtliche Winkel- 
werthe, die von mir angegeben wurden, bei diesem Salze sowohl, als bei den früheren, sind je das Mittel. aus sechszig Messungen.

Ferner wurden alle den oben angeführten schwefelselensauren Doppelsalzen entsprechenden selenschwefelsauren dargestellt und untersucht.

Zum Schlusse sei mir noch gestattet, eine kurze Uebersicht der einzelnen Resultate dieser Arbeit zu geben :

I. Zur Darstellung der Selensäure wird eine möglichst einfache und sichere Methode angegeben.

II. Versuch zur Darstellung des Selensäureanhydrids. Erhalten ein Gemenge von Selensäureanhydrid mit seleniger Säure.

III. Es ist möglich, Alaune herzustellen, in denen entweder alle an Kali gebundene Schwefelsäure, oder alle an Thonerde u. s. w. gebundene durch Selensäure ersetzt ist.

IV. Ebendasselbe gelingt auch bei den Doppelsalzen der allgemeinen Formel $\mathrm{m}_{2} \mathrm{RO}_{4} \cdot \mathrm{MRO}_{4}+6 \mathrm{H}_{2} \mathrm{O}$.

V. Es ist möglich, in allen diesen Salzen die Schwefelsäure Molecul für Molecul zu ersetzen durch Selensäure, und die Unmasse von Salzen, die so entstehen könnten, ist isomorph mit den Endgliedern der Reihe, den rein schwefelsauren und rein selensauren Doppelsalzen.

Erlangen, im April 1873. 